\title{
UNDERSTANDING AND ENHANCING POLARIZATION IN COMPLEX MATERIALS
}

\author{
Recent advances in theoretical methods and high-performance computing allow for \\ reliable first-principles investigations of complex materials. This article focuses on \\ calculating and predicting the properties of piezoelectrics and "designing" new materials \\ with enhanced piezoelectric responses. The authors consider two systems: boron-nitride \\ nanotubes (BNNTs) and polymers in the polyvinylidene fluoride (PVDF) family.
}

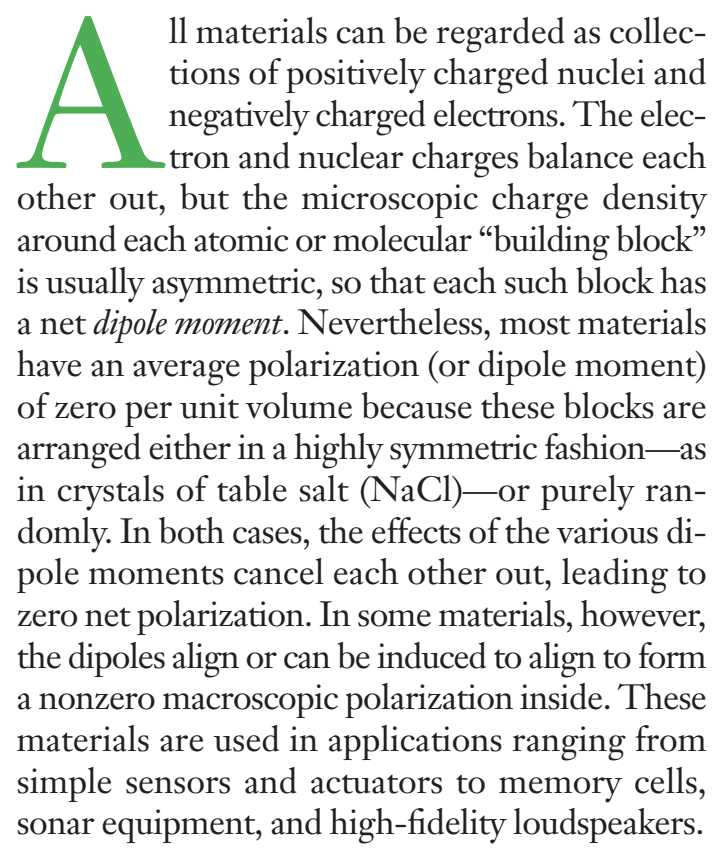

$1521-9615 / 04 / \$ 20.00$ ๑ 2004 IEEE

Copublished by the IEEE CS and the AIP

Jerry Bernholc, SERge M. NAKHManson,

and Marco BuONGIORNo Nardelli

North Carolina State University

VINCENT MEUNIER

Oak Ridge National Laboratory
Piezoelectrics are the most common materials that possess useful polarization-related properties. In such systems, applied stress leads to the emergence of a substantial electric field inside the material. Conversely, applying an external electric field to the material produces strain and a consequent volume change (usually elongation or contraction). In both cases-that is, under applied stress or an electric field-the positive and negative charges in the system are displaced differently, generating nonzero dipole moments and, thus, electric polarization. Though a piezoelectric can exhibit no polarization in its unperturbed state, a subclass of materials known as pyroelectrics (from the Greek word for "fire," the prefix pyro- indicates the permanent electric moment present in these systems) stays polar even without an external electric field or applied stress. These peculiar properties are usually associated with the reduced symmetry in these materials' geometrical structures, which prohibits their internal dipole fields' exact cancellation. Finally, there exist certain (quite rare) families of pyroelectric materials in which the polarization direction can be "switched" (for example, rotated by 180 degrees) by strain or poling in an external electric field. The unique properties of these ferroelectric materials, which often have large piezoelectric coefficients, make them extremely attractive candidates for numerous applications involving precise control and switching - the hallmark of advanced engineering. 
The remarkable properties of piezoelectric materials can be reliably reproduced, and even predicted, through large-scale calculations based on advanced quantum-mechanical concepts-without any experimental input. Researchers can thus use computers to "invent" novel, advanced materials designed for specific applications.

\section{Quantum Mechanics of Polarization}

The distribution of atoms and their electrons determines a material's electric field. To support an electric field, the material must be an insulator (otherwise, the field would be screened by electrons). For a given distribution of atomic nuclei, density-functional theory ${ }^{1}$ (DFT) accurately predicts electron distribution obtained from oneelectron wave functions $\psi_{i}$, which satisfy the Schrödinger-like equations (in Rydbergs):

$$
\left\{\begin{array}{l}
-\nabla^{2}+V_{N}(\mathbf{r})+\int \frac{2 \rho\left(\mathbf{r}^{\prime}\right) \mathrm{d} \mathbf{r}^{\prime}}{\left|\mathbf{r}-\mathbf{r}^{\prime}\right|} \\
+\mu_{x c}[\rho(\mathbf{r})]
\end{array}\right\} \psi_{i}(\mathbf{r})=\varepsilon_{i} \psi_{i}(\mathbf{r}),
$$

where $i=1, \ldots, M$.

Here, the first term represents the kinetic energy; the second is the potential due to all nuclei; the third is the classical electron-electron repulsion potential; and the fourth, the so-called exchangeand-correlation potential, accounts for the Pauli principle (which requires the wave function of the entire system to change sign when two electrons are exchanged) and spin effects. Summing over squares of the occupied wave functions gives the electron charge density $\rho(\mathbf{r})$. Although a real material contains a huge number of atoms, solid-state physics teaches us that many of the most important materials consist of periodically repeated unit cells that contain relatively few atoms. For example, both of our most important semiconductors, $\mathrm{Si}$ and GaAs, contain only two atoms in their smallest possible unit cells. More complex materials, including those containing defects, can also be modeled with large, periodically repeated unit cells containing tens or hundreds of atoms. The methods for solving DFT equations for such "supercells" are by now quite advanced; several techniques exist for obtaining the wave functions and even for optimizing a material's structure by minimizing the system's total energy. ${ }^{2}$ For example, the methods we use employ real-space grids and multigridacceleration techniques. ${ }^{3}$ The use of grids leads to effective parallelization over hundreds of CPUs through domain decomposition, thereby enabling accurate calculations for rather large supercells.

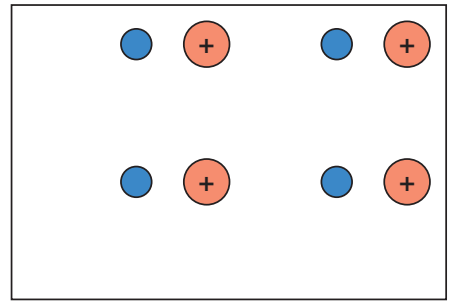

(a)

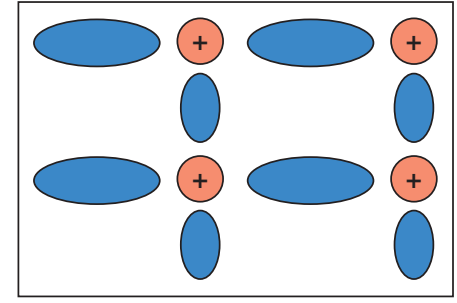

(b)
Figure 1. Schematic distribution of charges inside ferroelectric material. The red circles represent positive ions, and the blue circles and ovals depict the compensating electrons with negative charges. (a) The electrons are localized on negative ions, and the net electric field can be computed by simple summation over charges. (b) The electrons are more delocalized and form bonds between atoms. Because the summation over charges is not uniquely defined in this case, we must use more sophisticated Berry phase, or Wannier function, techniques to obtain the electric field.

In calculating polarization, however, the methodology is actually quite subtle because, in a polar material, the build-in field is compensated by charge buildup at its surfaces. Although the electric field per unit volume is well defined for a given structure and material, a straightforward summation over charges would have to include the entire sample and its surfaces, which is prohibitively expensive. In contrast, there are no surfaces in a periodic-unit-cell calculation, and the field must be zero to satisfy the periodic boundary conditions. Furthermore, we can use different unit cells to describe a periodic structure, and different cells cut through different bonds, thus leading to different dipole-moment values per unit cell. The dipole moment per unit cell is uniquely defined only for well-localized electrons-negative ions, for example. Figure 1 illustrates the distinction between the cases of well-localized and delocalized electrons forming bonds.

Nevertheless, even a periodic system's wave functions $\psi_{i}$ contain all the information required for an unequivocal computation of polarization. However, unlike the electron charge density $\rho(\mathbf{r})$, which is the squared modulus of the wave function (see the "Computing Polarization of a Periodic Solid" sidebar on p. 14), the polarization is fundamentally related to the wave function's phase. ${ }^{4,5}$ Let's assume that the system is adiabatically transformed (that is, without changing very slowly) from a nonpolar "reference" configuration to a polar one. During the transformation, an adiabatic current $\mathbf{j}$ must flow through the unit cell to account for the charge redistribution and the resulting polarization. Writing the complex wave functions as $|\Psi(\mathbf{r})| \exp \{i \varphi(\mathbf{r})\}$, 


\section{Computing Polarization of a Periodic Solid}

The polarization of a solid can be expressed as a sum of ionic and electronic contributions

$$
\mathbf{P}=\mathbf{P}_{\text {ion }}+\mathbf{P}_{e l}=\frac{1}{V_{\text {sample }}}\left[\sum_{\text {sample }} \sum_{l} e Z_{l} \mathbf{b}_{l}+\int_{\text {sample }} \mathbf{r} \rho(\mathbf{r}) d \mathbf{r}\right],
$$

where $Z_{l}$ and $\mathbf{b}_{l}$ are the ionic charge and position of the $l$-th atom in the solid, and $\rho(\mathbf{r})$ is the electron density.

For both the polar and the reference nonpolar configuration, the electronic polarization per unit cell is computed from the periodic parts of the solid's occupied wave functions, $\Psi_{n k}(\mathbf{r})=u_{n k}(\mathbf{r}) e^{i k r}$, as

$$
\mathbf{P}_{e l}=-\frac{2 i e}{(2 \pi)^{3}} \sum_{n \text { occ }} \int d \mathbf{k}\left\langle u_{n k}\left|\nabla_{k}\right| u_{n k}\right\rangle \text {. }
$$

A very useful alternative expression uses the so-called Wannier functions (WFs), which form an orthonormal basis and are defined as

$$
W_{n}(\mathbf{r})=V /(2 \pi)^{3} \int \Psi_{n k}(\mathbf{r}) d \mathbf{k}
$$

We can easily express the electron density in terms of WFs as

$$
\begin{aligned}
\rho(\mathbf{r}) & =-\frac{2 e}{(2 \pi)^{3}} \sum_{n \text { occ }} \int\left|\psi_{n k}(\mathbf{r})\right|^{2} d \mathbf{k} \\
& =-2 e \sum_{\mathbf{n} \text { occ }}\left|W_{n}(\mathbf{r})\right|^{2} \\
\mathbf{P}_{e l} & =\int \mathbf{r} \rho(\mathbf{r}) d \mathbf{r}=-2 e \sum_{n \text { occ }}\left\langle W_{n}|\mathbf{r}| W_{n}\right\rangle \\
& =-2 e \sum_{n \text { occ }} \mathbf{r}_{n},
\end{aligned}
$$

where $\mathbf{r}_{n}$ are the WF centers. Several algorithms can efficiently calculate Wannier functions. ${ }^{1,2}$

\section{References}

1. N. Marzari and D. Vanderbilt, "Maximally Localized Generalized Wannier Functions for Composite Energy Bands," Physical Rev. B, vol. 56, no. 20, 1997, pp. 12847-12865.

2. F. Gygi, J.-L. Fattebert, and E. Schwegler, "Computation of Maximally Localized Wannier Functions using a Simultaneous Diagonalization Algorithm," Computer Physics Comm., vol. 155, no. 1, 2003, pp. 1-6.

the current $\mathbf{j}=i\left(\Psi \nabla \Psi^{*}-\Psi^{*} \nabla \Psi\right)$ will depend on the phases $\varphi(\mathbf{r})$ of the system's wave functions. The geometric (or Berry) phase technique provides an elegant solution for computing electronic polarization in a periodic system by linking the phase evolution of the system's wave functions to the current flowing during the transformation and thus to the polarization change: $\Delta \mathbf{P}_{e l}=\int_{0}^{\Delta t} \mathbf{j}(t) d t$. We can compute the ionic part of polarization $\mathbf{P}_{\text {ion }}$ with a trivial summation over point charges.

If we denote by $\mathbf{G}_{i}$ the "reciprocal" (Fourier) lattice defined by $\mathbf{G}_{i} \cdot \mathbf{R}_{j}=2 \pi m$, where $\mathbf{R}_{j}$ belongs to the real-space lattice of all allowable translations of the unit cell, we can introduce the electronic (or Berry) phase for our system as $\varphi_{e l}=V_{\text {cell }} \mathbf{G}_{i} \cdot \mathbf{P}_{e l} / e$. By following the change in the Berry phase during the transformation (which must keep the system insulating), we can obtain the difference in polarization. However, because we calculate the Berry phase (as a true angular variable) modulo $2 \pi$, we can obtain the polarization only modulo $2 e \mathbf{R} / V_{\text {cell }}$. The difference in total polarization, $\Delta \mathbf{P}$, will then be well defined if $|\Delta \mathbf{P}|<\left|2 e \mathbf{R} / V_{\text {cell }}\right|$. The sidebar includes the expressions we used to evaluate $\mathbf{P}$ from the solid's periodic wave functions.

Alternatively, we can transform the periodic wave 
functions into "bonding" functions, which tend to be localized around individual atoms or bonds in the unit cells. A particular choice of such functions-namely, the Wannier functions (WFs), which form an orthonormal, localized basis-leads to a strikingly simple expression for polarization. As shown in the sidebar, the expression involves only the WF centers $\mathbf{r}_{n}=\left\langle W_{n}|\mathbf{r}| W_{n}\right\rangle$. We obtain $\Delta \mathbf{P}$ by simply treating these centers as point charges. ${ }^{6}$

\section{Searching for New Polar Materials}

The majority of existing ferroelectrics belong to two main families: those based on trimetal oxides containing lead, zirconium, and titanium (so-called PZT systems) and fluorine-based polymers, such as polyvinylidene fluoride (PVDF). The PZT ceramics are very strong piezo- and pyroelectrics; ${ }^{7}$ unfortunately, they're also brittle and quite heavy, and their lead content poses environmental problems. On the other hand, the piezoelectric polymers are lightweight, flexible, and virtually inert, but their polar properties are an order of magnitude weaker than those of PZTs. ${ }^{8}$ Materials that combined the attractive properties of both families would, of course, be very desirable. Using the reliable DFTbased methods ${ }^{2,3}$ for investigating materials and their properties, we thus embarked on a study of new materials and structures that could potentially exhibit much improved piezoelectric properties.

\section{Boron-Nitride Nanotubes}

Boron-nitride nanotubes (BNNTs) are tubular onedimensional systems with structures related to the well-known carbon nanotubes. Figure 2 shows the so-called zigzag nanotube's structure, in which planes intersecting the nanotube perpendicularly to its axis alternately contain boron and nitrogen atoms. Because the bond between the boron and nitrogen atoms is polar, there should be a potential for a sizable polar field along the tube's axis. In particular, model calculations using simplified quantum mechanical methods ${ }^{9}$ predict strong piezo- and pyroelectric effects and the spontaneous electric field that alternates in sign as the tubes' diameters increase. Given our interest in piezoelectric materials, we undertook an in-depth investigation of BNNTs and performed large-scale DFT-based calculations.

First, we used the Berry-phase approach to compute both the spontaneous polarization and piezoelectric constants for zigzag BNNTs, evaluating the contributions of the electrons and ions separately. For the latter, we performed a simple summation over the ionic charges, introducing an "ionic" phase analogous to the Berry phase for electrons:

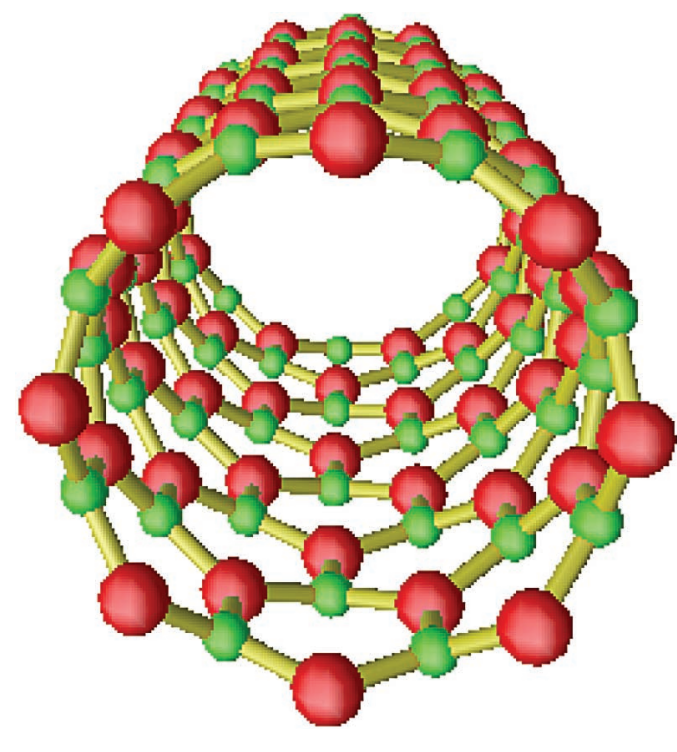

Figure 2. The structure of a boron-nitride nanotube. The atoms in the $\mathrm{BN}$ nanotube arrange themselves into a hexagonal network "rolled up" into a cylinder. The nanotube structure shown here is called "zigzag" because of the patterns formed by the atoms at the tube's edge.

$\varphi_{i o n}=V_{\text {cell }} \mathbf{G}_{k} \cdot \mathbf{P}_{\text {ion }} / e$. Because we need a nonpolar reference system to compute the change in spontaneous polarization, we created a nanotube composed of artificial atoms that were 50 percent $B$ and 50 percent $\mathrm{N}$, thus guaranteeing it to be nonpolar. We then smoothly changed the atoms' properties (computing polarization many times along the way) until the nanotube became a real system with 100 percent $\mathrm{B}$ or $\mathrm{N}$ atoms.

Figure 3a illustrates the ionic part of the spontaneous polarization in zigzag BNNTs. The inset shows the ionic phases translated into the $[-\pi, \pi]$ interval, which is the way they come out in the calculations, but the main graph shows the "unfolded" phases, obtained by eliminating the $2 \pi$ discontinuities and setting the nonpolar reference configuration's phase to zero. We see that the ionic phase is large and monotonically increasing. In fact, it is directly proportional to the zigzag nanotube's diameter, or the so-called nanotube index-the number of boron or nitrogen atoms along the tube circumference, in the case of a zigzag nanotube. In Figure 2, for example, the nanotube's index is $(8,0)$ because it has eight boron-nitrogen pairs of atoms along its zigzag edge. (The second index, " 0 ," indicates that the tube is of "zigzag" type.) For the unfolded phases, the ionic phase increases by $\pi / 3$ as the nanotube's 

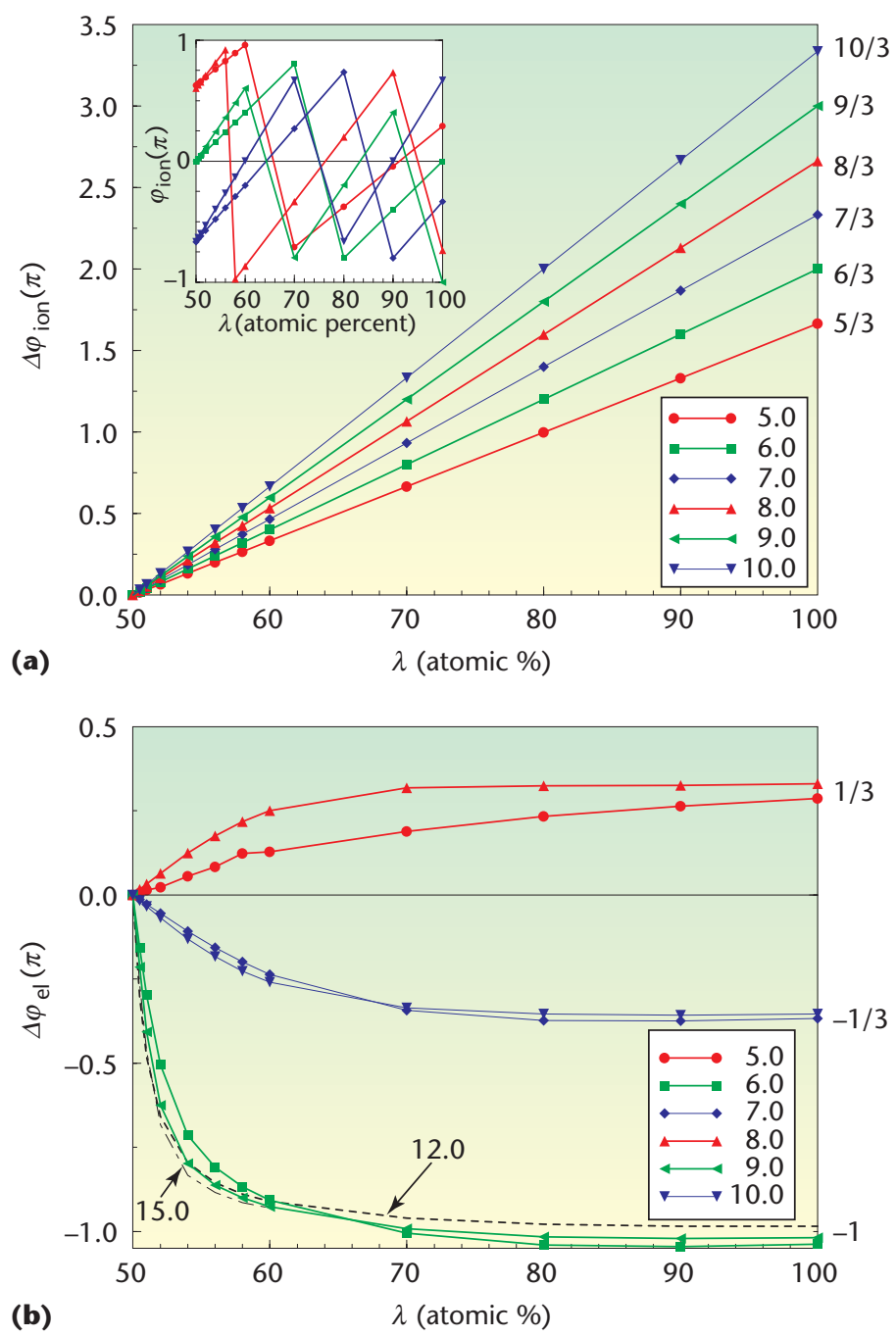

Figure 3. Ionic and electronic geometrical phases in zigzag $\mathrm{BN}$ nanotubes. (a) lonic-phase difference (in units of $\pi$ ) between the polar and nonpolar configurations of zigzag nanotubes grows monotonically with the nanotube index. The inset shows the ionic phases wrapped into the $[-\pi, \pi]$ interval. (b) Electronic-phase differences (in units of $\pi$ ) between the polar and nonpolar configurations for zigzag nanotubes take on three different constant values.

diameter increases - that is, as another hexagon is added around the tube's circumference-such that the total ionic phase for an $(n, 0) \mathrm{BN}$ nanotube becomes $n \pi / 3$.

For electronic phases, however, a DFT calculation gives only the reduced Berry phases (shown in Figure 3b). These look strikingly different from the reduced ionic phases and lack any obvious discontinuities that we could use to "unfold" them. Instead, there appear to be three classes with different $\Delta \varphi_{e l}$, depending on the zigzag tube's index: $\pi / 3$ for $n=3 l-1$,

$-\pi / 3$ for $n=3 l+1$, and

$-\pi$ for $n=3 l$,

where $l$ is an integer. The existence of three classes of behavior is surprising, however, given that the electronic charge density's ionic character (associated with the B-N bond) doesn't change with the nanotube index. We would expect the electronic part of the polarization to behave similarly to the ionic-that is, growing with the increasing nanotube index rather than taking on one of three constant values. However, we can't extract the unfolded phases from the Berry-phase calculations.

Actually, we gain a much more complete understanding of the intricate behavior of spontaneous polarization in zigzag BNNTs by using WFs. Because, in this case, we treat the electronic charges in exactly the same way as ionic charges (see the sidebar), we expected to find the same discontinuities in the curves for $\Delta \varphi_{e l}$, which would show us how to unfold these curves out of the $[-\pi, \pi]$ interval. Figure 4 summarizes our WF results, showing examples for $\mathrm{C}$ and $\mathrm{BN}$ zigzag nanotubes of any diameter; a schematic illustrates the shift of the Wannier centers in the adiabatic transformation from $\mathrm{C}$ to $\mathrm{BN}$. Because we can calculate the polarization from WF centers as

$$
\mathbf{P}_{e l}^{B N}=-\frac{2 e}{V} \sum_{i}\left(\mathbf{r}_{i}^{B N}-\mathbf{r}_{i}^{C}\right),
$$

the centers' shift is directly proportional to the BNNT's electronic polarization with respect to the nonpolar CNT.

Figure 4 shows the two classes of WFs that can contribute to the electronic polarization in BNNTs: $\sigma$ and $\pi$. The $\sigma$ WFs are centered in the middle of the $\mathrm{C}-\mathrm{C}$ bonds in carbon nanotubes, whereas they're shifted toward the anions (negatively charged ions-in this case, nitrogens) in BNNTs because of the $\mathrm{N}$ atoms' greater electronegativity. Because these shifts have the same magnitude along all three bond directions, the vector sum of all shifts is zero, and the $\sigma$ orbitals don't contribute to the system's total polarization. The $\pi$ WFs are centered on the anions in BNNTs, whereas they have a peculiar $\mathrm{V}$-shape in carbon nanotubes, with centers somewhat outside the C$\mathrm{C}$ bond. The sum of the $\pi$ Wannier centers' shifts is nonzero only for the axial component, which means the electronic polarization in BNNTs is purely axial and solely due to the $\pi$ orbitals, because $\sigma$ orbitals have a null net contribution.

The WF description's main advantage is that it allows for unambiguous unfolding of the elec- 
tronic phase. Analogous to the ionic phase, each individual hexagon carries a phase of $-\pi / 3$, leading to a total electronic phase of $-n \pi / 3$ for an $(n, 0)$ nanotube. This result demonstrates that the electronic polarization's direction in a BNNT is specified by the B-N bond's orientation and doesn't oscillate in direction with the nanotube diameter. We should point out that the WF results are completely consistent with the Berry-phase calculations: for any $n$, we can fold $\Delta \varphi_{e l}$ modulo $\pi$ instead of $2 \pi$, because the effective periodicity shown at the bottom of Figure 4 is $1 / 2 c$ instead of $c$. The electronic phase can thus be expressed in units of $-\pi / 3$, as described earlier.

When we combine the results for the ionic and electronic phases, we find the two contributions cancel exactly and that the total spontaneous polarization is zero-that is, the Wannier centers arrange themselves in a way that completely compensates the polarization due to ions. This general result is valid for BNNT nanotubes of any index ( $n$, $m) .{ }^{10}$ The only exceptions are nanotubes narrower than approximately $4 \AA$, where a residual polarization is present as an effect of the very high curvature. In such nanotubes, Wannier centers can't fully compensate the ionic polarization due to the atomic bonds' severe distortion, which makes these systems weakly pyroelectric. For example, $P=0.11$, 0.008 , and $0.002 \mathrm{C} / \mathrm{m}^{2}$ in $(3,1),(7,0)$, and $(12,0)$ nanotubes, respectively (with the positive direction for polarization being from anion to cation along the B-N bond).

The exact cancellation of the ionic and electronic terms is due to the screw-like arrangement of atoms of an isolated nanotube. However, nanotubes often aggregate into bundles because of van der Waals attraction, introducing symmetry-breaking terms and leading to weak pyroelectricity. For example, in $(7,0)$ bundles at equilibrium distance of $3.2 \AA$, polarization increases to $0.01 \mathrm{C} / \mathrm{m}^{2}$.

Turning to piezoelectric properties, the calculated piezoelectric constants for various bundles comprising zigzag BNNTs range from $0.39 \mathrm{C} / \mathrm{m}^{2}$ for $(5,0)$ tubes with $3.9 \AA$ Aiameter to $0.19 \mathrm{C} / \mathrm{m}^{2}$ for $10.2 \AA$ wide tubes. (We treat the tubes as quasi-1D objects, computing only the axial-tube stretching or squeezing-piezoelectric constants.) While these values are modest when compared with the constants of PZT-based materials, they're comparable with those of the piezoelectric polymers in current use. Because BN nanotubes are extremely strong and resilient, they could be prime candidates for applications requiring a combination of high mechanical strength, light weight, and substantial piezoelectric response.

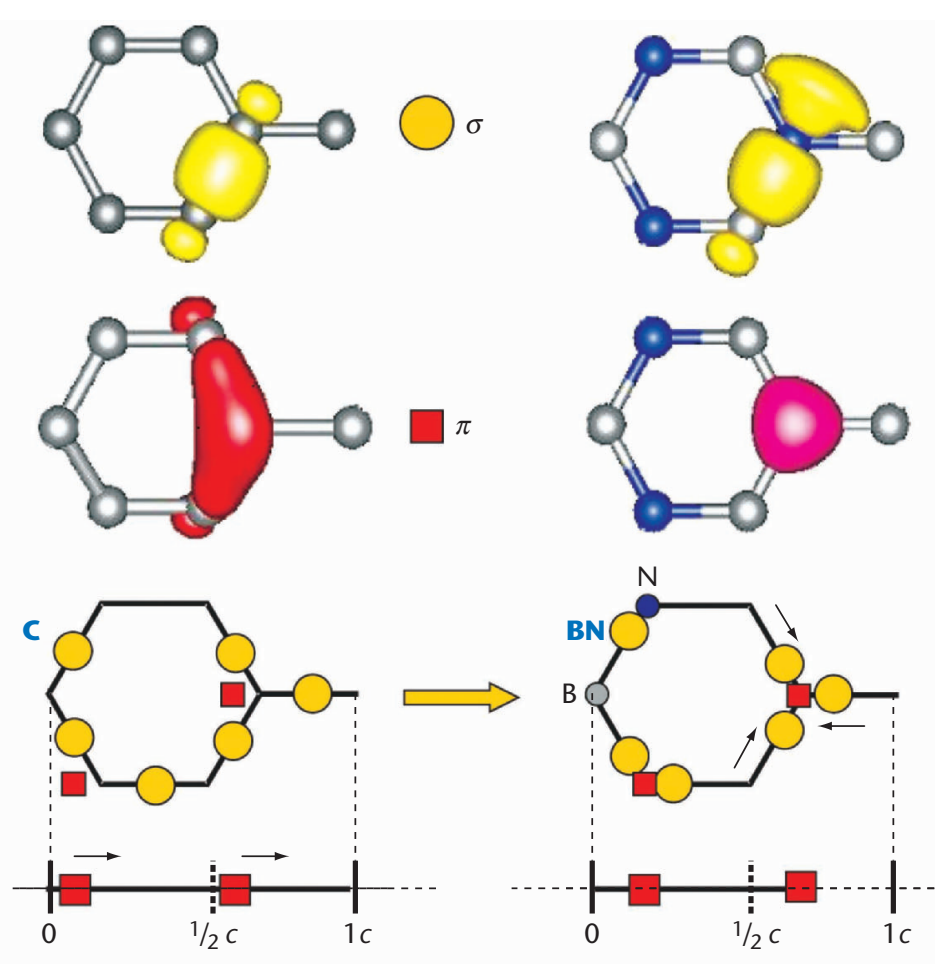

Figure 4. Examples of Wannier functions (WFs) of the $\sigma$ (s-like) and $\pi$ (plike) occupied bands of $C$ (left) and BN (right) nanotubes. The positions of the centers of $\sigma$ WFs are indicated by circles and those of $\pi$ by squares. The directions of the shifts of $\sigma$ and $\pi$ WFs in a transformation from $C$ to $B N$ are indicated by arrows. The vector sum of shifts of the $\sigma$ WFs is zero, so that the $\sigma$ WFs don't contribute to polarization. The bottom of the figure shows projections of the positions of $\pi$ WF centers onto the nanotube axis.

\section{Superpolar Polymers by Design}

Polymers based on PVDF, $\left[-\mathrm{CH}_{2}-\mathrm{CF}_{2}-\right]_{\mathrm{n}}$, are the most well-known and widely used family of polymer ferroelectrics. PVDF can be grown in a variety of phases that usually are only partially crystalline and partially disordered, which often degrades its useful properties. Figure 5 a shows the structure of PVDF's most polar $\beta$ phase. In this phase, the maximum polarization value arises from a highly ordered arrangement of intrinsically polar $\left[-\mathrm{CH}_{2}-\mathrm{CF}_{2}-\right]$ monomers. Each VDF monomer has a non-zero dipole moment-directed perpendicular to the carbon backbone- that is the sum of the F-C and C-H bonds' dipole moments. Figure 5 also shows the calculated values of spontaneous polarization in $\beta$-PVDF and related polymers.

Unfortunately, $\beta$-PVDF can currently be grown only $\sim 50$ percent crystalline, which dilutes its polar properties. However, PVDF copolymers with trifluoroethylene (TrFE) $\left[-\mathrm{CHF}-\mathrm{CF}_{2}-\right]$ and tetrafluoroethylene (TeFE) $\left[-\mathrm{CF}_{2}-\mathrm{CF}_{2}-\right]$, shown in 

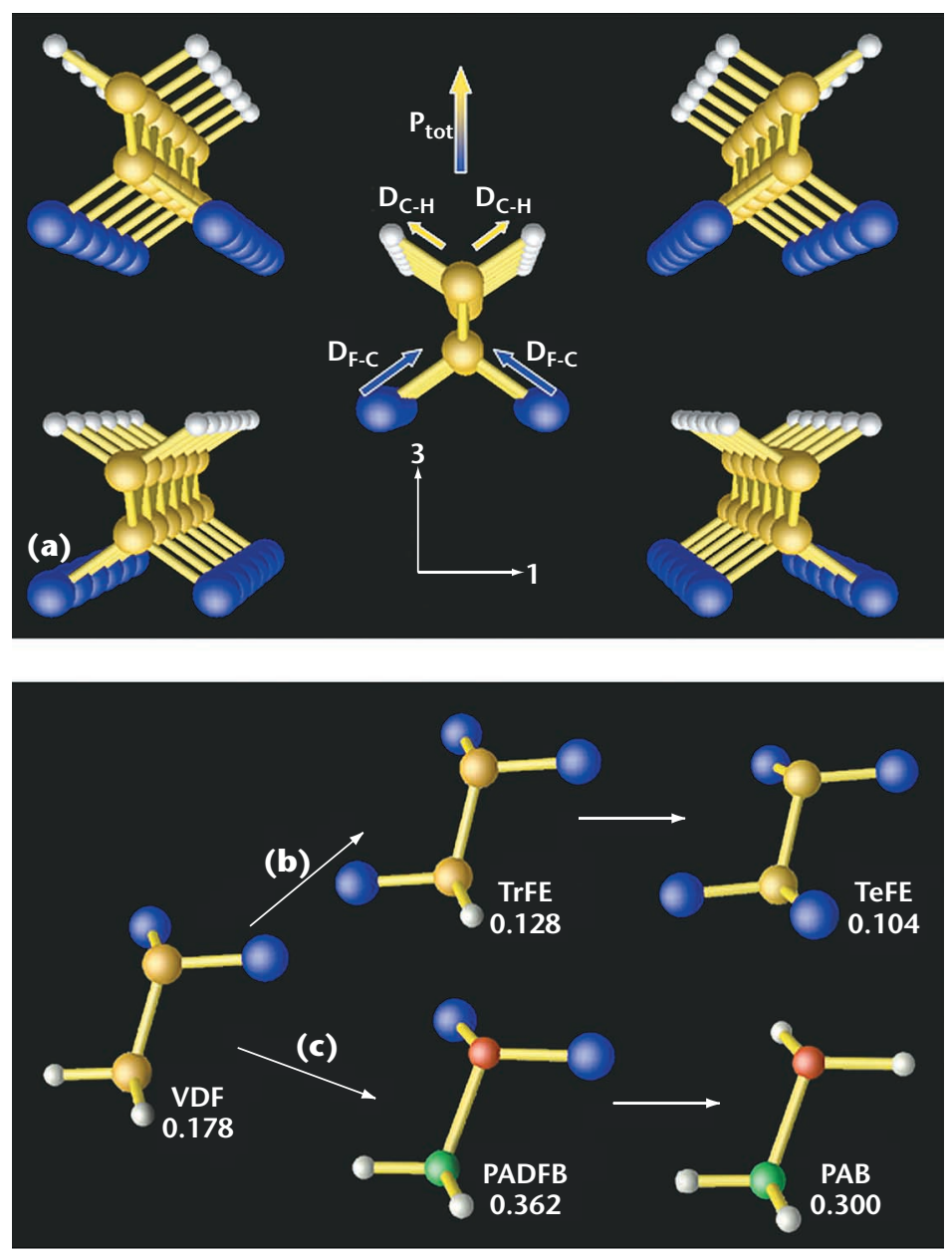

Figure 5. Evolution of polarization in $\beta$-PVDF and related polymers. (a) In this schematic of the structure of PVDF's crystalline $\beta$ phase, the F-C bond is far more ionic than the $\mathrm{C}-\mathrm{H}$ bond. This leads to polarization in the VDF monomer, directed perpendicular to the carbon backbone. $\mathrm{D}_{\mathrm{F}-\mathrm{C}}$ and $\mathrm{D}_{\mathrm{C}-\mathrm{H}}$ are the bond-dipole moments. (pathway $\mathrm{b}$ ) The VDF monomer evolves into TrFE and TeFE as the $\mathrm{H}$ atoms are substituted with $\mathrm{F}$ atoms. (pathway $\mathrm{c}$ ) The VDF monomer changes into polyaminodifluoroborane (PADFB) and polyaminoborane $(\mathrm{PAB})$ as the carbon backbone is substituted with boron and nitrogen atoms and $F$ atoms are substituted with hydrogens. The calculated spontaneous polarization for each polymer or copolymer is given in units of $\mathrm{C} / \mathrm{m}^{2}$.

Figure $5 \mathrm{~b}$, can be grown up to 90 percent crystalline. Because of their high crystallinity, these copolymers often exhibit better properties than "pure" $\beta$-PVDF, even though individual TrFE and TeFE monomers are less polar than VDF. Still, spontaneous polarization and piezoelectric response in all these materials are much weaker than in trimetal oxides, such as PZT. It would, of course, be highly beneficial to find a way to improve these polymers' polar properties, while retaining their superb mechanical properties and low environmental impact.
In fact, due to its simple structure, $\beta$-PVDF can serve as a "template" for the design of advanced polymer materials. We can use a simple "bonddipole" picture of polarization (as shown in Figure 5) to obtain clues about possible PVDF-like structures with improved polar properties. For example, we can make the fluorine end of the PVDF monomer more negative by replacing the carbon in the $\mathrm{F}-\mathrm{C}$ bonds with a less electronegative atom. In the same fashion, the hydrogen end of the monomer becomes more positive if a more electronegative atom than carbon participates in the hydrogen bonds.

Taking a hint from the close analogy between the $\mathrm{C}$ and BNNT structures, let's consider replacing the carbon backbone with boron nitride. If the $\mathrm{C}$ atom at the bottom of the monomer is replaced with $\mathrm{B}$ and the one at the top with $\mathrm{N}$, the polarity of both F-B and $\mathrm{N}-\mathrm{H}$ bonds should be magnified, thus enhancing the dipole moment per monomer. The resulting $\left[-\mathrm{BF}_{2}-\mathrm{NH}_{2}-\right]_{\mathrm{n}}$ polymer is called polyaminodifluoroborane (PADFB); an even simpler structure, $\left[-\mathrm{BH}_{2}\right.$ $\left.-\mathrm{NH}_{2}-\right]_{\mathrm{n}}$ polyaminoborane $(\mathrm{PAB})$ - the $\mathrm{BN}$ analogue of polyethylene-should become piezoelectric and pyroelectric. Figure $5 \mathrm{c}$ shows the monomers of both the PADFB and PAB structures.

Although this bond-dipole argument provides a potentially important suggestion for improving the properties of PVDF-like materials, it can neither reliably assess the magnitude of such improvements nor evaluate whether the improved material would be stable. The DFT-based methods for computing structural and polar properties, however, allow us to make accurate quantitative predictions about the proposed materials' stability as well as its improvement in polarization and piezoelectricity.

Table 1 presents the results of our calculations of spontaneous polarization and piezoelectricity for the PVDF polymer family and its BN-based analogs, ${ }^{11}$ together with comparisons to experimental data, where available. (Experimental results are extrapolated to 100 percent crystallinity and rescaled for the $75 / 25$ polymer/copolymer content where indicated.) All polymer models presented in the table are 100 percent crystalline. Coordinate axes for the piezoelectric stress tensor components $e_{\alpha \beta}$ (piezoelectric polarization $P_{\alpha}=\Sigma_{\beta} e_{\alpha \beta} \varepsilon_{\beta}$, where $\varepsilon_{\beta}$ is a strain along direction $\beta$ ) are shown in Figure 5 a with axis 2 being parallel to the polymer backbone. Spontaneous polarization $P_{3}^{s p}$ and piezoelectric constants $e_{3 \alpha}$ are given in units of $\mathrm{C} / \mathrm{m}^{2}$. In particular, we find a significant polarizing effect due to interactions between polymer chains. For isolated, individual PVDF chains, the dipole moment per monomer is 2 Debye (1 Debye $=3.336 \cdot 10^{-30}$ 
$\mathrm{C} \cdot \mathrm{m}$ ), but it increases to 3 Debye when fully aligned chains are assembled into $\beta$-PVDF crystal (see Figure 6). Unfortunately, pure $\beta$-PVDF's low crystallinity in currently available samples prevents a meaningful comparison with the data. However, comparing highly crystalline PVDF copolymers with experimental data shows excellent agreement with our calculations for both $\mathrm{TrFE}$ and TeFE structures. This effectively validates our approach to computing polarization in polymeric materials and provides credibility for our predictions regarding the "designed" BN-based polymers for which no experimental data is available.

As it turns out, the BN backbone leads to a substantial increase in polarization. For PADFB, it raises polarization by 100 percent compared to $\beta$ PVDF, while also creating a nearly twofold enhancement in piezoelectric properties. Realizing this increase experimentally would have a large technological impact. For $\mathrm{PAB}$, we obtain a more modest increase in spontaneous polarization, accompanied by an appropriate improvement in piezoelectricity.

An additional consideration in polymer design is stability of the ordered polar phase. Because the ordered phase is usually destroyed via random, thermally induced chain rotations, we computed the chain-rotation barriers for all the polymers discussed here. PAB, which doesn't contain fluorines, is significantly less stable than $\beta$-PVDF, with a low chain-rotation barrier of $0.28 \mathrm{eV}$ per monomer. The height of the barrier in PADFB is about $2.4 \mathrm{eV}$ per monomer-more than three times greater than in $\beta$-PVDF $(0.72 \mathrm{eV})$-indicating that the polar phase in PADFB should be much more stable than in $\beta$-PVDF.

It is surprising and encouraging that both of the BN-based polymers are already well-known, although to the best of our knowledge, no researchers have explored their polar properties. $\mathrm{PAB}$, for example, is routinely employed as a precursor for preparing hexagonal boron nitride. PA DFB has also been synthesized, although not in large quantities, but the literature suggests that developing such a process would be relatively straightforward. ${ }^{12}$ Furthermore, there is experimental evidence for PADFB's increased thermal stability. As grown, however, these polymers are unlikely to be ordered and, thus, highly ferro- and piezoelectric. In fact, the well-known PVDF-based polymers must be stretched and poled after growth to form ordered structures with nonzero macroscopic polarization. The increased chain-rotation barriers of BN-based polymers will make both the ordering and disordering transformations more
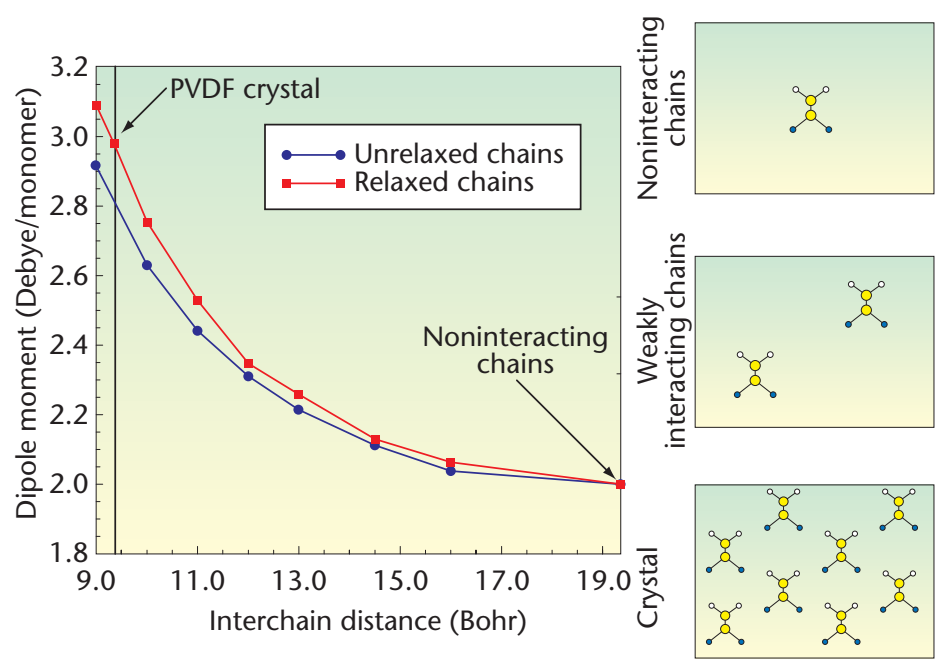

Figure 6. "Self-polarization" of $\beta$-PVDF chains. The blue curve corresponds to calculations with frozen ions (in which only the electronic charge is allowed to "relax"). The red curve corresponds to calculations in which both the electrons and ions are allowed to move. Comparing the curves shows that the major part of polarization enhancement comes from redistributing the electron charge as the chains are brought closer together; ionic movement plays only a minor role.

difficult, and advanced growth and processing methods might be required before highly polar samples can be produced. However, the large improvement in the ferro- and piezoelectric constants, combined with the excellent mechanical and environmental properties inherited from PVDF, makes these polymers highly attractive targets for future experimental studies and potential highvalue applications.

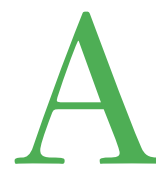
s we've illustrated, theoretical methods have now advanced to the point where accurate predictions of material properties, even for materials that do not yet exist, are both possible and practical. While density-functional theory provides the theoretical underpinning, studying the complex material structures that are so important in modern technology requires large computers. In our own calculations, for example, we used parallel IBM SP3 and Cray T3E supercomputers provided by the US Department of Defense's highperformance computing program, with typical running times of a few hours to a day on 16 to 64 CPUs, depending on the size of the system under investigation.

The recent progress in understanding the ferro- 
Table 1. Polar properties of the PVDF polymer family and the BN-based "structures by design."

\begin{tabular}{|c|c|c|c|c|c|}
\hline & $P_{3}^{s p}$ & $e_{31}$ & $e_{32}$ & $e_{33}$ & Comment \\
\hline$\beta$-PVDF & $0.178(0.131)$ & -0.268 & -0.270 & -0.332 & $\begin{array}{l}\text { Value in brackets: calculation with } \\
\text { noninteracting dipoles. }\end{array}$ \\
\hline $\mathrm{P}(\mathrm{VDF} / \mathrm{TrFE})(75 / 25)$ & $0.128(0.123)$ & -0.183 & -0.192 & -0.211 & $\begin{array}{l}\text { Value in brackets: polarization measured by } \\
\text { Tajitsu et al., see Japan J. Applied Physics, vol. } \\
26,4,1987 \text {, pp. 554-560. }\end{array}$ \\
\hline $\mathrm{P}(\mathrm{VDF} / \mathrm{TeFE})(75 / 25)$ & $0.104(0.118)$ & -0.135 & -0.145 & -0.150 & $\begin{array}{l}\text { Value in brackets: polarization measured by } \\
\text { Tasaka and Miyata, see J. Applied Physics, vol. } \\
57,3,1985 \text {, pp. } 906-910\end{array}$ \\
\hline PADFB & 0.362 & -0.493 & -0.580 & -0.555 & \\
\hline $\mathrm{PAB}$ & 0.300 & -0.348 & -0.398 & -0.431 & \\
\hline $\mathrm{PbTiO}_{3}$ crystal & 0.88 & -0.93 & & 3.23 & $\begin{array}{l}\text { Calculation (DFT/Berry phases), tetragonal } \\
\text { configuration with a polar (001) axis. See } \\
\text { reference } 7 \text {. }\end{array}$ \\
\hline$(7,0)$ BNNT & 0.008 & & & 0.293 & Polar direction is along the axis of the tube. \\
\hline
\end{tabular}

and piezoelectric properties of materials has been achieved by developing auxiliary methods for computing the difference in the quantum phase between polar and reference nonpolar configurations. However, an equivalent description in terms of WFs is more physically transparent, given that their centers behave as effective charge centers. Our two explicit examples, BNNTs and ferroelectric polymers, illustrate the ability to reliably calculate complex structures' polar properties and provide physically intuitive explanations for the calculated behavior, as well as concepts for predicting new materials with significantly enhanced properties. In addition to identifying sizable piezoelectric response in BNNTs and predicting a new class of ferroelectric polymers, with up to 100 percent improvement in ferro- and piezoelectric properties, we also succeeded with subtler predictions. For example, with detailed analysis of computational results for hypothetical polymer structures-a capability unique to theory and computational models - we predicted that interchain interaction leads to significant enhancement in polar properties and that the $\mathrm{BN}$ analogue of PVDF should be more stable at high temperatures than its C-based counterpart.

Coupled with exponentially expanding hardware capabilities, increasingly sophisticated theoretical methods and computational techniques will surely enhance the role of materials theory in satisfying technological needs. In the fairly near future, many materials will likely be designed on a computer, so that researchers will examine only a handful of final candidate structures in the laboratory, following a principles-based approach, rather than investigating various combinations of materials. While com- binatorial techniques are effective in examining many closely related compounds or mixtures, the materials world is far too rich and complex to fully explore via combinatorial means.

Our predictions of new ferroelectric polymers have already led to a collaboration with leading polymer researchers, who will attempt to grow them in ordered thin films, so that their polar properties can be measured. We are also collaborating with these researchers on other polymeric materials, which are based on PVDF but have better solvation properties and are thus easier to deposit. Our future work will aim at developing materials with significantly enhanced polar and dielectric properties, which should have a variety of applications in microelectronics and in power conditioning and storage.

\section{References}

1. R.M. Dreizler and E.K.U. Gross, Density Functional Theory, Springer, 1990.

2. J. Bernholc, "Computational Materials Science: The Era of Applied Quantum Mechanics," Physics Today, vol. 52, no. 9, 1999, pp. 30-35.

3. E.L. Briggs, D.J. Sullivan, and J. Bernholc, "A Real-Space Multigrid-Based Approach to Large-Scale Electronic Structure Calculations," Physical Rev. B, vol. 54, no. 20, 1996, pp. 1436214375.

4. R. Resta, "Macroscopic Polarization in Crystalline Dielectrics: The Geometric Phase Approach," Rev. Modern Physics, vol. 66, no. 3, 1994, pp. 899-915.

5. R.D. King-Smith and D. Vanderbilt, "Theory of Polarization in Crystalline Solids," Physical Rev. B, vol. 47, no. 3, 1993, pp. 1651-1654.

6. N. Marzari and D. Vanderbilt, "Maximally Localized Generalized Wannier Functions for Composite Energy Bands," Physical Rev. B, vol. 56, no. 20, 1997, pp. 12847-12865.

7. G. Sághi-Szabó, R.E. Cohen, and H. Krakauer, "First-Principles Study of Piezoelectricity in Tetragonal $\mathrm{PbTiO}_{3}$ and 
$\mathrm{PbZr}_{1 / 2} \mathrm{Ti}_{1 / 2} \mathrm{O}_{3}$," Physical Rev. B, vol. 59, no. 20, 1999, pp. 12771-12776

8. Ferroelectric Polymers: Chemistry, Physics and Applications, H.S. Nalwa, ed., Marcell Dekker, 1995.

9. E.J. Mele and P. Král, "Electric Polarization of Heteropolar Nanotubes as a Geometric Phase," Physical Rev. Letters, vol. 88, no. 5,2002, pp. 056803-056806.

10. S.M. Nakhmanson et al., "Spontaneous Polarization and Piezoelectricity in Boron Nitride Nanotubes," Physical Rev. B, vol. 67, no. 23, 2003, pp. 235406-235410.

11. S.M. Nakhmanson, M. Buongiorno Nardelli, and J. Bernholc, "Ab Initio Studies of Polarization and Piezoelectricity in Vinylidene Fluoride and BN-Based Polymers," Physical Rev. Letters, vol. 92, no. 11, 2004, pp. 115504-115507.

12. S.Y. Pusatcioglu et al., "Thermal Stability and Molecular Weight of Two New Boron-Nitrogen Polymers," J. Applied Polymer Science, vol. 21, no. 6, 1977, pp. 1561-1567.

Jerry Bernholc is the Drexel Professor of Physics at North Carolina State University, where he serves as director of the Center for High-Performance Simulations. $\mathrm{He}$ 's also a visiting distinguished scientist at Oak Ridge National Laboratory. His recent work focuses on multigrid, multiscale, and linear-scaling methods in nanoscale physics. He received a BS in physics and mathematics and a PhD in physics from the University of Lund, Sweden. He is a recipient of IBM's Outstanding Innovation Award, NCSU Alumni's Outstanding Research Award, NSF's Creativity Award, and the American Physical Society's Beams Award. Contact him at bernholc@ ncsu.edu; nemo.physics.ncsu.edu/ bernholc.

Serge M. Nakhmanson was a postdoctoral research associate at North Carolina State University, where he studied the polarization-related properties of nanostructures and crystalline and polymeric materials using multigrid calculation techniques. He received a BS and an MSc in physics from St. Petersburg State University, Russia, and a PhD in condensed-matter theory from Ohio University in Athens, Ohio. He recently moved to Rutgers University. Contact him at nakhmans@ physics.rutgers.edu.

Marco Buongiorno Nardelli is an assistant professor of physics at North Carolina State University and an $R \& D$ staff member in the Computer Science and Mathematics Division at Oak Ridge National Laboratory. His research interests focus on the application of $\mathrm{ab}$ initio electronic structure techniques to the theoretical study of important aspects of the physics of materials. He received a PhD in condensed-matter theory from the International School for Advanced Studies (SISSA/ISAS) in Trieste, Italy. He is a recipient of the Sigma Xi Faculty Research Award, NCSU Chapter. Contact him at mbnardelli@ncsu.edu.

Vincent Meunier is an R\&D staff member in the Computer Science and Mathematics Division at Oak Ridge
National Laboratory. His main activities are focused on first-principles studies of nanoscopic materials, including quantum transport phenomena and electronic processes governing the formation of scanning-probe microscopy images. Meunier received a BS in physics, MSc in physics and chemistry, and a $\mathrm{PhD}$ in physics from the University of Namur (FUNDP), Belgium. Contact him at meunierv@ornl.gov; www.csm.ornl.gov/ccsg.

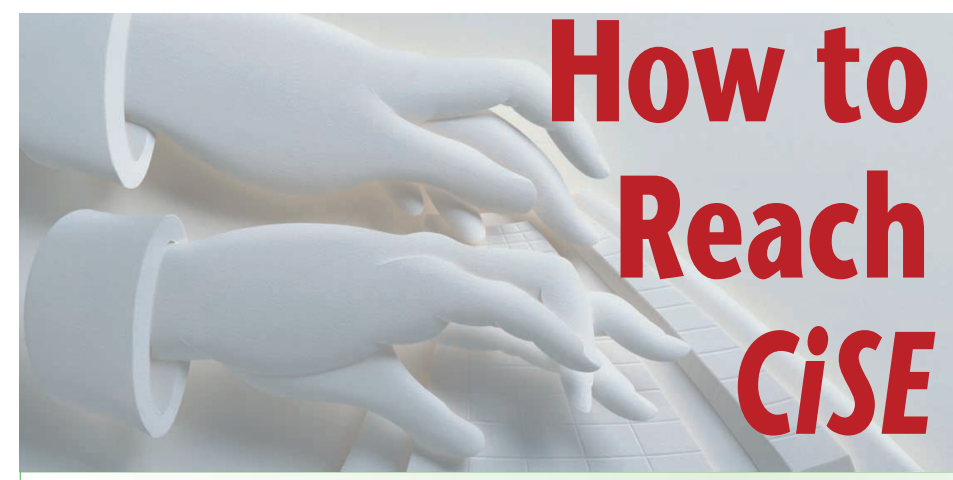

\section{Writers}

For detailed information on submitting articles, write to cise@computer.org or visit www.computer.org/cise/author.htm.

\section{Letters to the Editors}

Send letters to Jenny Ferrero, Contact Editor, jferrero@computer.org. Please provide an email address or daytime phone number with your letter.

\section{On the Web}

Access www. computer.org/cise/ or http://cise.aip.org for information about CiSE.

\section{Subscription Change of Address (IEEE/CS)}

Send change-of-address requests for magazine subscriptions to address.change@ieee.org. Be sure to specify CiSE.

\section{Subscription Change of Address (AIP)}

Send general subscription and refund inquiries to subs@aip.org.

\section{Subscribe}

Visit https://www.aip.org/forms/journal_catalog/order_form_fs.html or www.computer.org/subscribe/.

\section{Missing or Damaged Copies}

If you are missing an issue or you received a damaged copy (IEEE/CS), contact membership@computer.org. For AIP subscribers, contact kgentili@aip.org.

\section{Reprints of Articles}

For price information or to order reprints, send email to cise@computer.orgor fax+1 7148214010.

\section{Reprint Permission}

To obtain permission to reprint an article, contact William Hagen, IEEE Copyrights and Trademarks Manager, atcopyrights@ieee.org. 